\title{
Development of the Mayo Investigational Neuromodulation Control System: toward a closed-loop electrochemical feedback system for deep brain stimulation:
}

Laboratory investigation

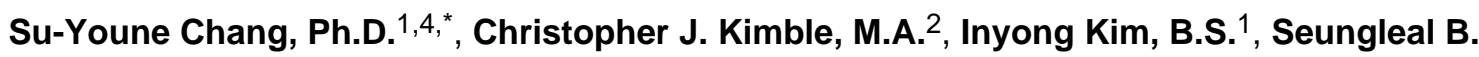
Paek, B.S. ${ }^{1}$, Kenneth R. Kressin, B.S. ${ }^{2}$, Joshua B. Boesche, B.S., M.B.A. ${ }^{2}$, Sidney V. Whitlock, B.S. ${ }^{2}$, Diane R. Eaker, M.S. ${ }^{2}$, Aimen Kasasbeh, M.D., Ph.D. ${ }^{1}$, April E. Horne, B.S., M.B.A. ${ }^{2}$, Charles D. Blaha, Ph.D. ${ }^{3}$, Kevin E. Bennet, B.Sc.Ch.E., M.B.A. ${ }^{2}$, and Kendall H. Lee, M.D., Ph.D. ${ }^{1,4}$

${ }^{1}$ Department of Neurologic Surgery, Mayo Clinic, Rochester, Minnesota

2Division of Engineering, Mayo Clinic, Rochester, Minnesota

${ }^{3}$ Department of Psychology, University of Memphis, Tennessee

${ }^{4}$ Department of Physiology and Biomedical Engineering, Mayo Clinic, Rochester, Minnesota

\begin{abstract}
Object-Conventional deep brain stimulation (DBS) devices continue to rely on an open-loop system in which stimulation is independent of functional neural feedback. The authors previously proposed that as the foundation of a DBS "smart" device, a closed-loop system based on neurochemical feedback, may have the potential to improve therapeutic outcomes. Alterations in neurochemical release are thought to be linked to the clinical benefit of DBS, and fast-scan cyclic voltammetry (FSCV) has been shown to be effective for recording these evoked neurochemical changes. However, the combination of FSCV with conventional DBS devices interferes with the recording and identification of the evoked analytes. To integrate neurochemical recording with neurostimulation, the authors developed the Mayo Investigational Neuromodulation Control System (MINCS), a novel, wirelessly controlled stimulation device designed to interface with FSCV performed by their previously described Wireless Instantaneous Neurochemical Concentration Sensing System (WINCS).
\end{abstract}

(CAANS, 2013

Address correspondence to: Kendall H. Lee, M.D., Ph.D., Departments of Neurologic Surgery and Physiology and Biomedical Engineering, Mayo Clinic, 200 First St. SW, Rochester, MN 55905. lee.kendall@ mayo.edu.

* Dr. Chang and Mr. Kimble contributed equally to this work.

Disclosure

Support was provided to K.H.L. by the NIH (Grant Nos. K08 NS 52252, R01 NS 70872, and R01 NS 74013) and The Grainger Foundation. The authors report no conflict of interest concerning the materials or methods used in this study or the findings specified in this paper.

Author contributions to the study and manuscript preparation include the following. Conception and design: Lee, Chang, Kimble, Blaha, Bennet. Acquisition of data: Chang, Kim, Paek, Kasasbeh. Analysis and interpretation of data: Chang, Kim, Paek, Kasasbeh. Drafting the article: Chang, Kimble, Kasasbeh. Critically revising the article: Chang, Kimble, Kasasbeh, Blaha. Reviewed submitted version of manuscript: Lee, Chang, Kimble, Blaha, Bennet. Approved the final version of the manuscript on behalf of all authors: Lee. Administrative/technical/material support: Kimble, Kressin, Boesche, Whitlock, Eaker, Horne, Bennet. Study supervision: Lee. 
Methods-To test the functionality of these integrated devices, various frequencies of electrical stimulation were applied by MINCS to the medial forebrain bundle of the anesthetized rat, and striatal dopamine release was recorded by WINCS. The parameters for FSCV in the present study consisted of a pyramidal voltage waveform applied to the carbon-fiber microelectrode every 100 msec, ramping between $-0.4 \mathrm{~V}$ and $+1.5 \mathrm{~V}$ with respect to an $\mathrm{Ag} / \mathrm{AgCl}$ reference electrode at a scan rate of either $400 \mathrm{~V} / \mathrm{sec}$ or $1000 \mathrm{~V} / \mathrm{sec}$. The carbon-fiber microelectrode was held at the baseline potential of $-0.4 \mathrm{~V}$ between scans.

Results-By using MINCS in conjunction with WINCS coordinated through an optic fiber, the authors interleaved intervals of electrical stimulation with FSCV scans and thus obtained artifactfree wireless FSCV recordings. Electrical stimulation of the medial forebrain bundle in the anesthetized rat by MINCS elicited striatal dopamine release that was time-locked to stimulation and increased progressively with stimulation frequency.

Conclusions-Here, the authors report a series of proof-of-principle tests in the rat brain demonstrating MINCS to be a reliable and flexible stimulation device that, when used in conjunction with WINCS, performs wirelessly controlled stimulation concurrent with artifact-free neurochemical recording. These findings suggest that the integration of neurochemical recording with neurostimulation may be a useful first step toward the development of a closed-loop DBS system for human application.

\section{Keywords}

deep brain stimulation; dopamine; fast scan cyclic voltammetry; wireless device; functional neurosurgery

Deep brain stimulation (DBS) is an established treatment for essential tremor, dystonia, and Parkinson's disease ${ }^{5,12,35}$ and is an emerging therapysion, addiction, and obsessivecompulsive disorder. $6,16,19,25$ Despite extensive clinical applications, the therapeutic benefit of DBS is limited by conventional stimulation devices that operate independently of physiological and/or neurochemical feedback. In these conventional open-loop systems, stimulation parameters are determined in an imprecise, trial-and-error manner based on the patient's subjective experience of clinical benefit at the time of stimulator programming. Such open-loop systems cannot adapt to ongoing fluctuations in neurophysiological functions.

Because neurochemical release from the output nuclei at the stimulation site is thought to be linked to the clinical benefit of DBS, $8,18,20,26,27$ we previously proposed the use of neurochemical information as the recording feedback component of a DBS "smart" device. ${ }^{23,24}$ Stimulation parameters in such a closed-loop device would be controlled by continuous neurochemical feedback, which would, in turn, keep neurochemical concentrations at optimal levels.

As a first step in this process, we developed the Wireless Instantaneous Neurochemical Concentration Sensing System (WINCS) to conduct wireless fast-scan cyclic voltammetry (FSCV) recordings of DBS-evoked neurochemical release, and we confirmed the system's functionality in rodents and a large animal model (pig). ${ }^{7,32,33}$ Briefly, FSCV is based on cyclic voltammetry, a classic electrochemical technique, and provides real-time and 
chemically resolved neurochemical measurements in the brain. The most common recording electrode used for analyte detection is a carbon-fiber microelectrode (CFM), which offers a relatively broad voltage range in aqueous solutions. By using FSCV at CFMs, changes in the extracellular concentration of electroactive molecules, such as dopamine, norepinephrine, serotonin, and adenosine, as well as other physiologically relevant agents (for example, oxygen), can be monitored. ${ }^{38}$

Applied for the first time in human patients undergoing DBS surgery for essential tremor, WINCS-based FSCV recorded and quantified changes in adenosine release upon DBS electrode insertion. ${ }^{10}$ These changes in adenosine were concurrent with marked reduction in tremor amplitude, supporting the hypothesis that neurochemical release plays a role in the therapeutic mechanisms of DBS. However, it is important to note that once the DBS stimulator was activated, the applied electrical pulses markedly interfered with the FSCV signal, producing stimulus artifacts that hindered the recording and identification of the analytes detected.

To enable reliable analyte recording and identification of stimulation-induced neurochemical changes evoked by DBS, we have developed a wirelessly controlled electrical neurostimulator, the Mayo Investigational Neuromodulation Control System (MINCS), as a prototype for future development of a human closed-loop neurostimulation system. To synchronize stimulation with FSCV recordings, MINCS is connected to WINCS by an optical fiber. By interleaving intervals of stimulation with FSCV scans, stimulus artifacts in the recorded data are eliminated. While conventional stimulators and electrochemical instruments have been successfully synchronized for the study of central neurotransmission in animal research, $36,37,39,40$ the unified software that controls both WINCS and MINCS enhances the versatility with which synchronized stimulation can be performed. Here we report a series of proof-of-principle tests in the rat brain demonstrating MINCS to be a reliable and flexible stimulation device that, when used in conjunction with WINCS, performs wirelessly controlled stimulation concurrent with artifact-free neurochemical recording. The development of these complementary prototypes represents the first steps toward the development of a closed-loop, fully integrated DBS system for the treatment of patients with neurological and psychiatric diseases.

\section{Methods}

\section{Instrumentation}

WINCS-Based Wireless FSCV—As previously described, ${ }^{7,10,32,33}$ WINCS combines FSCV with wireless telemetry to enable the detection of neurochemicals at a CFM implanted in the brain of a human or animal. The WINCS hardware incorporates front-end analog circuitry for FSCV, a microcontroller, and a Bluetooth radio, packaged on a single multilayer printed circuit board. The WINCS is powered by a rechargeable lithium-ion battery. Electrode current is converted to voltage by a trans-impedance amplifier. A difference amplifier subtracts the overriding FSCV voltage waveform, produced by a digital-to-analog converter (DAC) incorporated in the microcontroller and then applied to the CFM by feedback action through the transimpedance amplifier. ${ }^{7}$ An analog-to-digital converter (ADC) in the microcontroller samples the output of the difference amplifier at 
rates as high as 100,000 samples per second. This data stream is transmitted by Bluetooth to the base station computer, to be recorded, analyzed, and displayed by custom software, WincsWare, developed by the Mayo Clinic Division of Engineering. ${ }^{21}$ WincsWare has now been refined to integrate wireless control of electrical stimulation by MINCS.

The parameters for FSCV in the present study consisted of a pyramidal voltage waveform applied to the CFM every $100 \mathrm{msec}$, ramping between $-0.4 \mathrm{~V}$ and $+1.5 \mathrm{~V}$ with respect to an $\mathrm{Ag} / \mathrm{AgCl}$ reference electrode at a scan rate of either $400 \mathrm{~V} / \mathrm{sec}$ or $1000 \mathrm{~V} / \mathrm{sec}$. The duration of each scan was $9.5 \mathrm{msec}$ at a scan rate of $400 \mathrm{~V} / \mathrm{sec}$ and $3.8 \mathrm{msec}$ at a scan rate of $1000 \mathrm{~V} /$ sec. The CFM was held at the baseline potential of $-0.4 \mathrm{~V}$ between scans. In addition to making electrochemical measurements at a CFM with subsecond temporal resolution and submillimeter spatial resolution, FSCV data, presented in the form of a backgroundsubtracted voltammogram, facilitates identification of the chemical origin of the signal. ${ }^{28,30}$

CFM for In Vivo FSCV Recordings-A CFM was constructed by attaching a single polyacrylonitrile-based carbon fiber (7- $\mu$ m diameter, Cytec) to a nitinol (an alloy of nickel and titanium) extension wire with a silver-based conductive paste developed by our laboratory. The connection between the carbon fiber and the nitinol wire was covered with polyamic acid (Sigma-Aldrich) and heated to $200^{\circ} \mathrm{C}$ to polymerize the polyamic acid into a polyimide film. The exposed carbon fiber was trimmed under a dissecting microscope to a length of approximately $50 \mu \mathrm{m} .{ }^{10} \mathrm{An} \mathrm{Ag} / \mathrm{AgCl}$ reference electrode was prepared by exposing 0.5 mmofa 31-gauge Teflon-coated silver wire (A-M Systems, Inc.) and chlorinating the exposed tip in saline with a $9-\mathrm{V}$ dry cell battery. ${ }^{15}$

MINCS-The MINCS is a wirelessly controlled neurostimulator, which was designed to synchronize stimulation with the WINCS FSCV recording to eliminate stimulus artifact during electrochemical recording. As shown in Fig. 1, the MINCS circuit board and battery are packaged in a plastic case. The WincsWare software application, running on a Windows personal computer base station, enables the user to modify stimulation parameters and to control MINCS stimulation remotely. The MINCS incorporates a 32-bit ARM-core mixedsignal microcontroller (Stellaris LM3S9B96, Texas Instruments), front-end analog circuitry for electrical stimulation, circuitry for sensing and fault protection, and a Bluetooth transceiver, all packaged on a multilayer printed circuit board and powered by a rechargeable, 6.5-Watt-hour lithium-ion battery (Ultralife). The sensor for the optical interface with WINCS is attached to the MINCS case. As a prototype for a future human-use stimulator, MINCS incorporates fault detection and protection mechanisms intended to ensure patient safety in the event of a malfunction.

As directed by the base station, the MINCS microcontroller controls the hardware to produce the stimulus sequences defined by the operator. The microcontroller manages communication with the base station via Bluetooth, monitors stimulus voltage and current, reports measurements to the base station, and performs various diagnostic and calibration functions. The microcontroller's 10-bit ADC is used to measure the applied stimulus voltage and current, and to periodically verify battery capacity. 
To produce a stimulation sequence, the microcontroller adjusts a 14-bit DAC and directs its output signal to either a voltage-regulated or current-regulated driver, each based on the LM8261 operational amplifier (National Semiconductor). An array of analog switch integrated circuits steers the stimulus pulses emitted by the amplifier to the selected electrodes. The output stimulation range is $50 \mathrm{mV}$ to $10 \mathrm{~V}$ in voltage-regulated mode and 10 $\mu \mathrm{A}$ to $10 \mathrm{~mA}$ in current-regulated mode, assuming adequate compliance for the electrode impedance. The typical impedance of a Medtronic 3389 DBS lead in tissue ranges from 410 to $4000 \Omega$. The minimum pulse duration is $50 \mu \mathrm{sec}$, and the maximum is $2 \mathrm{msec}$. Pulse patterns can be either monophasic or biphasic, and the applied charge can be asymmetrically balanced. The operator can select the polarity of the electrode leads. To improve accuracy, an automated calibration procedure performed during production compensates for offsets, variances, and internal impedances in the analog signal path. A second calibration procedure improves the accuracy of the ADC that measures stimulus voltage and current.

The National Semiconductor LMX9838 Bluetooth serial-port module, also used by WINCS, is a Federal Communications Commission-licensed, single-chip Bluetooth 2.0 implementation. Crystal and antenna are both integrated. Bluetooth technology operates in the unlicensed Industrial, Scientific and Medical (ISM) band at 2.400-2.485 GHz, using a spread-spectrum, frequency-hopping, full-duplex signal.

WincsWare-WincsWare, the in-house designed software used in this study, simultaneously controls both the WINCS and MINCS. As shown in Fig. 2, WincsWare allows the operator to define MINCS stimulus pulse amplitude, frequency, duration, polarity, and pulse-train pattern. Custom-designed stimulus sequences can be defined by stringing together 50- $\mu$ sec pulses in an arbitrary manner to deliver monophasic or biphasic waveforms that include pyramid, sawtooth, charge-balanced biphasic, and sync burst pulses. The MINCS can also select various stimulation modes and presentation characteristics, such as initial delay and pulse pattern settings. It can direct stimulation to any combination of the 4 separate electrode contacts found in standard clinical DBS electrodes (for example, Medtronic DBS lead models 3387 and 3389). In addition, the stimulator can measure applied voltage and load current, enabling the base station software to calculate electrode impedance in vivo, thus providing the user with information about the integrity of each electrode contact.

Stimulation can be precisely interleaved with WINCS FSCV scans by means of a sync signal transmitted through an optical fiber connecting the 2 devices (Fig. 1) WincsWare facilitates the process of selecting stimulus pulse durations and repetition rates, which permits seamless synchronization of stimulation with FSCV scans, as defined by the chosen FSCV parameters. WincsWare advises the user when practical constraints are violated (for example, when the stimulus sequence to be interleaved exceeds the duration of the intervals between FSCV scans).

To optimize FSCV for the detection of dopamine, serotonin, adenosine, histamine, or oxygen, the various parameters are fully adjustable (for example, baseline voltage, positive and negative peak potentials, sample rate, and scan and repetition rates). ${ }^{9,17,32}$ As shown in Fig. 3, WincsWare displays a dynamic "color plot" of electrode current (represented by 
horizontal color traces) as a function of applied potential (vertical axis), for a sequence of FSCV scans over time (horizontal axis). Also displayed are 2D plots of electrode current versus applied potential for individual FSCV scans, and a strip chart of electrode currents measured at selected applied potentials. User controls enable background correction and filtering of the data displayed in these plots. Intervals of stimulation are depicted in a separate strip chart. All stimulation is time stamped and synchronized with FSCV data in the recorded data files, which may be played back using WincsWare, or exported for processing by third-party software. The user can insert time-stamped annotations into the recorded file, as data collection proceeds. The plots shown in Fig. 3 correspond to a representative example of the magnitude and time course of medial forebrain bundle (MFB) stimulationevoked dopamine release in the striatum of an anesthetized rat.

\section{In Vivo Experiments}

Animal Surgery-The feasibility and functionality of the synchronized MINCS and WINCS devices were tested in vivo by measuring changes in striatal dopamine release evoked by electrical stimulation in 3 adult male Sprague-Dawley rats weighing 300-400 g. The rats were housed under standard conditions (12-hour/12-hour light/dark cycle; lights on at 6:00 a.m.) with ad libitum access to food and water. Care was provided in accordance with National Institutes of Health guidelines and was approved by the Mayo Clinic Institutional Animal Care and Use Committee.

The rats were anesthetized with urethane (1.6 g/ $/ \mathrm{kg}$ intraperitoneally) and immobilized in a commercially available stereotactic head frame (David Kopf Instruments). Three 1-mmdiameter holes were drilled through the skull of each rat to accommodate implantation of an $\mathrm{Ag} / \mathrm{AgCl}$ reference electrode that served as the counter auxiliary electrode, a stimulating electrode (twisted bipolar electrode; Plastics One, MS 303/2), and a CFM that served as the working electrode. Stereotactic coordinates were obtained from a rat brain atlas ${ }^{29}$ based on a flat-skull position using bregma and dura as reference points with coordinates anteroposterior (AP), mediolateral (ML), and dorsoventral (DV). The stimulating electrode was placed just above the MFB (in mm: AP $-4.6, \mathrm{ML}+1.2$, and DV -8.0 to -9.0 ) of the right hemisphere. The CFM was positioned ipsilaterally in the dorsomedial striatum (in $\mathrm{mm}$ $\mathrm{AP}+1.2, \mathrm{ML}+2.0$, and $\mathrm{DV}-4.5$ to -6.0 ). The reference electrode was inserted $0.5 \mathrm{~mm}$ into superficial cortical tissue contralateral to the stimulating and recording electrodes. The positions of the stimulating and recording electrodes were adjusted prior to the experiment to obtain a robust signal for electrically evoked dopamine release measured in the striatum. Once the signal was determined to be optimal, the electrodes' positions were not changed for the remainder of the experiment.

\section{Comparison of Electrical Stimulation With and Without Synchronization-To}

test the ability of MINCS to eliminate stimulation-induced interference in detecting neurochemicals released in vivo, we compared the responses of MFB striatal dopamine release evoked by MINCS stimulation and recorded by WINCS FSCV with the responses obtained with a conventional, hardwired stimulation device (Isolated Pulse Stimulator Model 2100, A-M) in individual rats. 
MINCS Functionality Tests-The functionality of MINCS was also tested in a separate experiment by examining the effects of increasing stimulation frequency on MFB stimulation-evoked striatal dopamine release in the rat. Stimulations (120 biphasic pulses at 30,60 , or $120 \mathrm{~Hz}$ ) were separated by 5 minutes and were repeated 3 times for each test stimulation frequency.

\section{Results}

\section{Electrical Stimulation and FSCV With and Without Synchronization}

Figure 4 shows a representative example of the effects of electrical stimulation and neurochemical recordings with and without synchronization. The integration of MINCS with WINCS was effective in delivering electrical stimulation $(60-\mathrm{Hz}, 200-\mu \mathrm{A}, 2-\mathrm{msec}$ biphasic pulses for 30 seconds) to the rat MFB and recording stimulation-evoked changes in dopamine release in the rat striatum and did so without generating stimulus pulse artifacts. As shown in Fig. 4A, using a conventional stimulator, unsynchronized stimulus pulses periodically would coincide with an FSCV scan and thus interfere with FSCV recordings. As shown in Fig. 4B however, MINCS permitted continuous interleaving of the applied series of electrical stimulus pulses with the applied series of WINCS-based FSCV scans.

Examination of the color plot recorded during conventional stimulation (Fig. 4C) reveals stimulus pulse artifacts that appear as repeating diagonal bands. In the color plot depicting MINCS stimulation (Fig. 4D) such artifacts are completely absent. As shown in Fig. 4E, a stimulus pulse that coincided with an FSCV scan resulted in a marked distortion of the cyclic voltammogram compared with voltammograms recorded with synchronized stimulation (Fig. 4F). Distortion of the recorded voltammograms by coincident stimulus pulses resulted in negative-going spike artifacts that are readily apparent in the time course of stimulation-evoked changes in dopamine release (Fig. 4G). As shown in Fig. 4E, these artifacts are entirely absent during MINCS synchronized stimulation. It is important to note that with a much briefer stimulation period (for example, 5 seconds) both the magnitude and temporal pattern of stimulation-evoked dopamine release would have been significantly disrupted by the stimulus artifact.

\section{Striatal Dopamine Release as a Function of Stimulation Frequency}

To further assess the functionality of MINCS, various frequencies of electrical stimulation were applied to the MFB of the anesthetized rat, and striatal dopamine release was recorded by WINCS. The number of stimulations (120 biphasic pulses), stimulation intensity (200 $\mu \mathrm{A})$ and pulse width $(2 \mathrm{msec})$ were fixed, and the duration of the stimulus sequence was adjusted to compensate for changes in frequency. For example, stimulus sequence duration was 4 seconds at $30 \mathrm{~Hz}, 2$ seconds at $60 \mathrm{~Hz}$, and 1 second at $120 \mathrm{~Hz}$. These varied stimulus sequences were continuously monitored on an oscilloscope (PDS8102T, Owon) to confirm that they were correctly delivered to the animal. Figure 5 shows the effects of stimulation frequency on striatal dopamine release in a representative rat. Consistent with previous reports, ${ }^{15,22}$ increasing the stimulation frequency from $30 \mathrm{~Hz}$ to $120 \mathrm{~Hz}$ resulted in a progressive increase in the magnitude of striatal dopamine release as measured here by 
WINCS-based FSCV (Fig. 5D; mean \pm SEM of 3 stimulations/frequency; $30 \mathrm{~Hz}, 2.09 \pm$ $0.31 \mathrm{nA} ; 60 \mathrm{~Hz}, 12.45 \pm 0.23 \mathrm{nA}$; and $120 \mathrm{~Hz}, 16.05 \pm 1.26 \mathrm{nA}$ ).

\section{Discussion}

For more than 2 decades, interleaving electrical stimulus pulses during the quiet intervals between FSCV scans using alternating current-powered conventional stimulators and electrochemical instruments under software control has proven an effective means of eliminating stimulus pulse artifacts in animal studies. ${ }^{36,37,39,40}$ Food and Drug Administration-approved battery-powered implantable pulse generators for human DBS were not designed to integrate with FSCV; they are incapable of interleaving stimulus pulses with FSCV scans and therefore cannot eliminate the artifacts that interfere with analyte detection and identification. ${ }^{10}$ To demonstrate artifact-free wireless FSCV recordings of DBS-evoked neurochemical release, we developed a versatile, functionally integrated, wirelessly controlled system capable of delivering electrical stimulation concurrently with in vivo neurochemical recording. The central innovation of this system is its coupling of battery-powered, fault-protected electrical stimulation with WINCS-based FSCV via an optical synchronization signal. The system is controlled by WincsWare, custom software that also facilitates the programming of MINCS stimulation parameters for seamless integration with WINCS-based FSCV. The synchronization of stimulation with FSCV effectively eliminated stimulation-induced artifacts, thereby enabling the reliable recording and identification of an electroactive analyte (dopamine). Electrical stimulation of the MFB in the anesthetized rat by MINCS elicited striatal dopamine release that was time-locked to stimulation and increased progressively with stimulation frequency.

In addition to producing traditional step-pulse waveforms over a wide range of stimulation intensities, frequencies, and pulse widths, MINCS can generate a variety of user-designed stimulation waveforms. This capability may prove invaluable in exploring the potential therapeutic effects of novel stimulation parameters on various neurological and psychiatric disorders, as well as on forebrain arousal regulation in the severely injured brain. ${ }^{4-6,12,16,19,25,31,35}$ Potentially, custom stimulation waveforms could enable highly selective activation of neuronal populations and provide more robust stimulation effects. ${ }^{1,2,13}$ In addition, in various DBS applications, such individualized waveforms could reduce energy consumption and increase the battery life of the DBS pulse generator. ${ }^{14}$ Finally, the fact that MINCS and WINCS are wirelessly controlled suggests that the integrated system may have potential for long-term neurochemical monitoring. Wireless chronic monitoring in animals and eventually in humans would allow subjects the freedom to move and behave naturally without the restrictions imposed by wired devices. ${ }^{3}$

Although further study is needed, the successful integration of MINCS-based stimulation with WINCS-based FSCV holds promise for the development of a closed-loop system in which feedback from an implanted neurochemical microsensor drives DBS to maintain neurochemical release at optimal levels. At present, DBS parameters (for example, amplitude, frequency, pulse width, and electrode contacts) can be independently programmed postsurgically, but their optimal configuration is dependent on the DBS programmer's experience and can only be determined in response to patient reports of 
improved benefit. This trial-and-error open-loop programming system has been in place for more than 20 years. It is necessarily inexact and can be time-consuming and costly. ${ }^{11,34} \mathrm{It}$ has been proposed that an integrated DBS system could, in the near future, serve as an adjunct surgical tool, providing neurochemical feedback to help guide electrode placement during DBS surgery. ${ }^{23,24}$ In the longer term, an implantable integrated closed-loop DBS system that relies on objective neurochemical feedback, rather than on subjective experience, could have a profound effect on enhancing clinical outcomes. The successful coupling of stimulation and neurochemical recording reported here can be considered a device appropriate for human use.

\section{Conclusions}

The results of this proof-of-principle study demonstrate the functionality of a novel wirelessly controlled stimulation device (MINCS). Synchronized with a wirelessly controlled neurochemical recording device (WINCS), it enabled artifact-free, stimulationevoked, in vivo FSCV recordings of changes in striatal dopamine in the rat brain. These initial findings suggest that the combination of MINCS and WINCS has the potential to advance our understanding of the neurochemical mechanisms underlying the therapeutic effectiveness of DBS, with implications for future development of a closed-loop neuromodulation system. Such a system could serve as an intraoperative tool to improve the precision of electrode targeting. A fully implantable system could improve DBS programming by dynamically adjusting stimulation parameters in response to neurochemical feedback, thus markedly enhancing clinical benefit.

\section{Acknowledgments}

We acknowledge the technical support of Dr. Hoon-ki P. Min and Mr. Michael P. Marsh and the editorial support of Dr. Penelope Duffy and Ms. Emily Knight.

\section{Abbreviations used in this paper}
ADC
analog-to-digital converter
CFM
carbon-fiber microelectrode
DAC
digital-to-analog converter
DBS
deep brain stimulation
FSCV
fast-scan cyclic voltammetry
MFB
medial forebrain bundle
MINCS Mayo Investigational Neuromodulation Control System
WINCS Wireless Instantaneous Neurochemical Concentration Sensing System

\section{References}

1. Agarwal R, Sarma SV. The effects of DBS patterns on basal ganglia activity and thalamic relay: a computational study. J Comput Neurosci. 2012; 33:151-167. [PubMed: 22237601]

J Neurosurg. Author manuscript; available in PMC 2014 December 01. 
2. Agarwal R, Sarma SV. Restoring the basal ganglia in Parkinson's disease to normal via multi-input phase-shifted deep brain stimulation. Conf Proc IEEE Eng Med Biol Soc. 2010; 2010:1539-1542. [PubMed: 21096376]

3. Arfin SK, Long MA, Fee MS, Sarpeshkar R. Wireless neural stimulation in freely behaving small animals. J Neurophysiol. 2009; 102:598-605. [PubMed: 19386759]

4. Benabid AL. What the future holds for deep brain stimulation. Expert Rev Med Devices. 2007; 4:895-903. [PubMed: 18035954]

5. Benabid AL, Chabardes S, Mitrofanis J, Pollak P. Deep brain stimulation of the subthalamic nucleus for the treatment of Parkinson's disease. Lancet Neurol. 2009; 8:67-81. [PubMed: 19081516]

6. Benabid AL, Torres N. New targets for DBS. Parkinsonism Relat Disord. 2012; 18 (Suppl 1):S21S23. [PubMed: 22166437]

7. Bledsoe JM, Kimble CJ, Covey DP, Blaha CD, Agnesi F, Mohseni P, et al. Development of the Wireless Instantaneous Neurotransmitter Concentration System for intraoperative neurochemical monitoring using fast-scan cyclic voltammetry. Technical note. J Neurosurg. 2009; 111:712-723. [PubMed: 19425890]

8. Carron R, Chabardès S, Hammond C. Mechanisms of action of high-frequency deep brain stimulation. A review of the literature and current concepts. Neurochirurgie. 2012; 58:209-217. (Fr). [PubMed: 22465140]

9. Chang SY, Jay T, Muñoz J, Kim I, Lee KH. Wireless fast-scan cyclic voltammetry measurement of histamine using WINCS-a proof-of-principle study. Analyst. 2012; 137:2158-2165. [PubMed: 22416270]

10. Chang SY, Kim I, Marsh MP, Jang DP, Hwang SC, Van Gompel JJ, et al. Wireless fast-scan cyclic voltammetry to monitor adenosine in patients with essential tremor during deep brain stimulation. Mayo Clin Proc. 2012; 87:760-765. [PubMed: 22809886]

11. Cooper S, Bowes M. Surgical considerations for tremor and dystonia. Cleve Clin J Med. 2012; 79 (Suppl 2):S40-S43. [PubMed: 22761270]

12. Deuschl G, Raethjen J, Hellriegel H, Elble R. Treatment of patients with essential tremor. Lancet Neurol. 2011; 10:148-161. [PubMed: 21256454]

13. Feng XJ, Shea-Brown E, Greenwald B, Kosut R, Rabitz H. Optimal deep brain stimulation of the subthalamic nucleus-a computational study. J Comput Neurosci. 2007; 23:265-282. [PubMed: 17484043]

14. Foutz TJ, McIntyre CC. Evaluation of novel stimulus waveforms for deep brain stimulation. J Neural Eng. 2010; 7:066008. [PubMed: 21084732]

15. Garris PA, Christensen JR, Rebec GV, Wightman RM. Real-time measurement of electrically evoked extracellular dopamine in the striatum of freely moving rats. J Neurochem. 1997; 68:152161. [PubMed: 8978721]

16. Greenberg BD, Rauch SL, Haber SN. Invasive circuitry-based neurotherapeutics: stereotactic ablation and deep brain stimulation for OCD. Neuropsychopharmacology. 2010; 35:317-336. [PubMed: 19759530]

17. Griessenauer CJ, Chang SY, Tye SJ, Kimble CJ, Bennet KE, Garris PA, et al. Wireless Instantaneous Neurotransmitter Concentration System: electrochemical monitoring of serotonin using fast-scan cyclic voltammetry-a proof-of-principle study. Laboratory investigation. J Neurosurg. 2010; 113:656-665. [PubMed: 20415521]

18. Hammond C, Ammari R, Bioulac B, Garcia L. Latest view on the mechanism of action of deep brain stimulation. Mov Disord. 2008; 23:2111-2121. [PubMed: 18785230]

19. Holtzheimer PE, Mayberg HS. Deep brain stimulation for psychiatric disorders. Annu Rev Neurosci. 2011; 34:289-307. [PubMed: 21692660]

20. Johnson MD, Miocinovic S, McIntyre CC, Vitek JL. Mechanisms and targets of deep brain stimulation in movement disorders. Neurotherapeutics. 2008; 5:294-308. [PubMed: 18394571]

21. Kimble CJ, Johnson DM, Winter BA, Whitlock SV, Kressin KR, Horne AE, et al. Wireless Instantaneous Neurotransmitter Concentration Sensing System (WINCS) for intraoperative neurochemical monitoring. Conf Proc IEEE Eng Med Biol Soc. 2009; 2009:4856-4859. [PubMed: 19963865] 
22. Kuhr WG, Bigelow JC, Wightman RM. In vivo comparison of the regulation of releasable dopamine in the caudate nucleus and the nucleus accumbens of the rat brain. J Neurosci. 1986; 6:974-982. [PubMed: 3486259]

23. Lee, KH.; Blaha, CD.; Bledsoe, JM. Mechanisms of action of deep brain stimulation: a review. In: Krames, ES.; Peckham, PH.; Rezai, AR., editors. Neuromodulation. Burlington, MA: Academic Press; 2009. p. 157-169.

24. Lee KH, Blaha CD, Garris PA, Mohseni P, Horne AE, Bennet KE, et al. Evolution of deep brain stimulation: human electrometer and smart devices supporting the next generation of therapy. Neuromodulation. 2009; 12:85-103. [PubMed: 20657744]

25. Maciunas RJ, Maddux BN, Riley DE, Whitney CM, Schoenberg MR, Ogrocki PJ, et al. Prospective randomized double-blind trial of bilateral thalamic deep brain stimulation in adults with Tourette syndrome. J Neurosurg. 2007; 107:1004-1014. [PubMed: 17977274]

26. McIntyre CC, Grill WM, Sherman DL, Thakor NV. Cellular effects of deep brain stimulation: model-based analysis of activation and inhibition. J Neurophysiol. 2004; 91:1457-1469. [PubMed: 14668299]

27. McIntyre CC, Savasta M, Walter BL, Vitek JL. How does deep brain stimulation work? Present understanding and future questions. J Clin Neurophysiol. 2004; 21:40-50. [PubMed: 15097293]

28. Michael DJ, Joseph JD, Kilpatrick MR, Travis ER, Wightman RM. Improving data acquisition for fast-scan cyclic voltammetry. Anal Chem. 1999; 71:3941-3947. [PubMed: 10500480]

29. Paxinos, G.; Watson, C. The Rat Brain in Stereotaxic Coordinates. 4. New York: Academic Press; 1998.

30. Robinson DL, Venton BJ, Heien ML, Wightman RM. Detecting subsecond dopamine release with fast-scan cyclic voltammetry in vivo. Clin Chem. 2003; 49:1763-1773. [PubMed: 14500617]

31. Schiff ND. Moving toward a generalizable application of central thalamic deep brain stimulation for support of forebrain arousal regulation in the severely injured brain. Ann N Y Acad Sci. 2012; 1265:56-68. [PubMed: 22834729]

32. Shon YM, Chang SY, Tye SJ, Kimble CJ, Bennet KE, Blaha CD, et al. Comonitoring of adenosine and dopamine using the Wireless Instantaneous Neurotransmitter Concentration System: proof of principle. Laboratory investigation. J Neurosurg. 2010; 112:539-548. [PubMed: 19731995]

33. Shon YM, Lee KH, Goerss SJ, Kim IY, Kimble C, Van Gompel JJ, et al. High frequency stimulation of the subthalamic nucleus evokes striatal dopamine release in a large animal model of human DBS neurosurgery. Neurosci Lett. 2010; 475:136-140. [PubMed: 20347936]

34. Siddiqui MS, Haq IU, Okun MS. Deep brain stimulation in movement disorders. Continuum (Minneap Minn). 2010; 16 (1 Movement Disorders):110-130. [PubMed: 22810183]

35. Welter ML, Grabli D, Vidailhet M. Deep brain stimulation for hyperkinetics disorders: dystonia, tardive dyskinesia, and tics. Curr Opin Neurol. 2010; 23:420-425. [PubMed: 20610993]

36. Wiedemann DJ, Garris PA, Near JA, Wightman RM. Effect of chronic haloperidol treatment on stimulated synaptic overflow of dopamine in the rat striatum. J Pharmacol Exp Ther. 1992; 261:574-579. [PubMed: 1533666]

37. Wiedemann DJ, Kawagoe KT, Kennedy RT, Ciolkowski EL, Wightman RM. Strategies for low detection limit measurements with cyclic voltammetry. Anal Chem. 1991; 63:2965-2970. [PubMed: 1789456]

38. Wightman RM. Detection technologies. Probing cellular chemistry in biological systems with microelectrodes. Science. 2006; 311:1570-1574. [PubMed: 16543451]

39. Zimmerman JB, Kennedy RT, Wightman RM. Evoked neuronal activity accompanied by transmitter release increases oxygen concentration in rat striatum in vivo but not in vitro. J Cereb Blood Flow Metab. 1992; 12:629-637. [PubMed: 1618942]

40. Zimmerman JB, Wightman RM. Simultaneous electrochemical measurements of oxygen and dopamine in vivo. Anal Chem. 1991; 63:24-28. [PubMed: 1810167] 


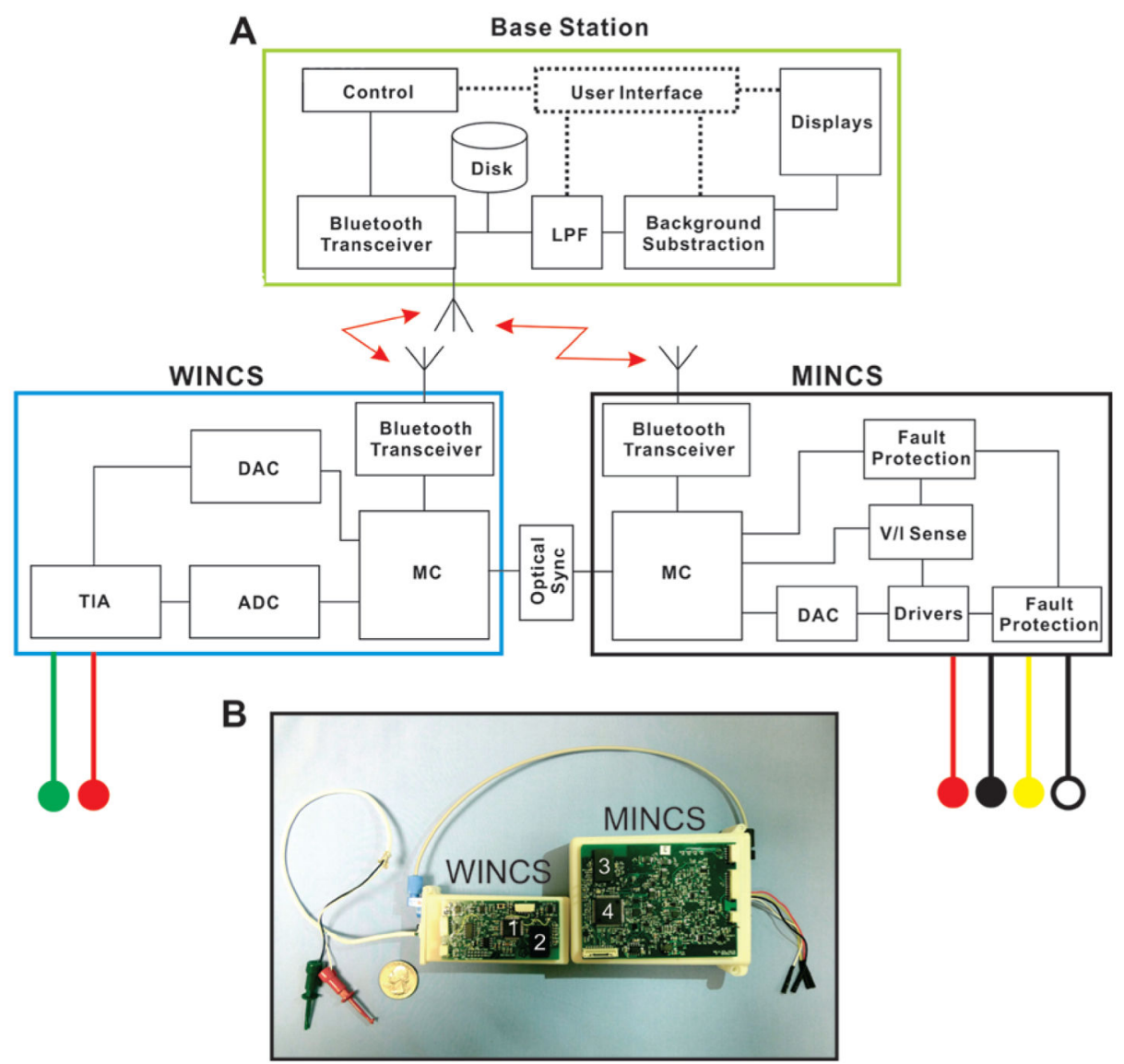

Fig. 1.

MINCS-WINCS hardware. A: Schematic representation of the computer base station, WINCS, and MINCS. The neurochemical recording device, WINCS, and the synchronized stimulator, MINCS, are linked via an optical connection. Both units connect wirelessly via Bluetooth technology to a base station, which commands both systems via WincsWare software. Leads from each device provide connections to the recording and reference electrodes (red and green circles) and 4 stimulating electrodes (red, black, yellow, and white circles). B: Photograph of the MINCS-WINCS hardware showing relative size, optical connection, and recording and stimulating electrode leads. $\mathrm{ADC}=$ analog-to-digital converter; DAC $=$ digital-to-analog converter; $\mathrm{LPF}=$ lowpass filter; $\mathrm{MC}=$ microcontroller; TIA $=$ transimpedance amplifier; $\mathrm{V} / \mathrm{I}$ Sense $=$ voltage/current sense. Numbers 1 and 4 indicate the microcontrollers; 2 and 3 are the Bluetooth modules. 


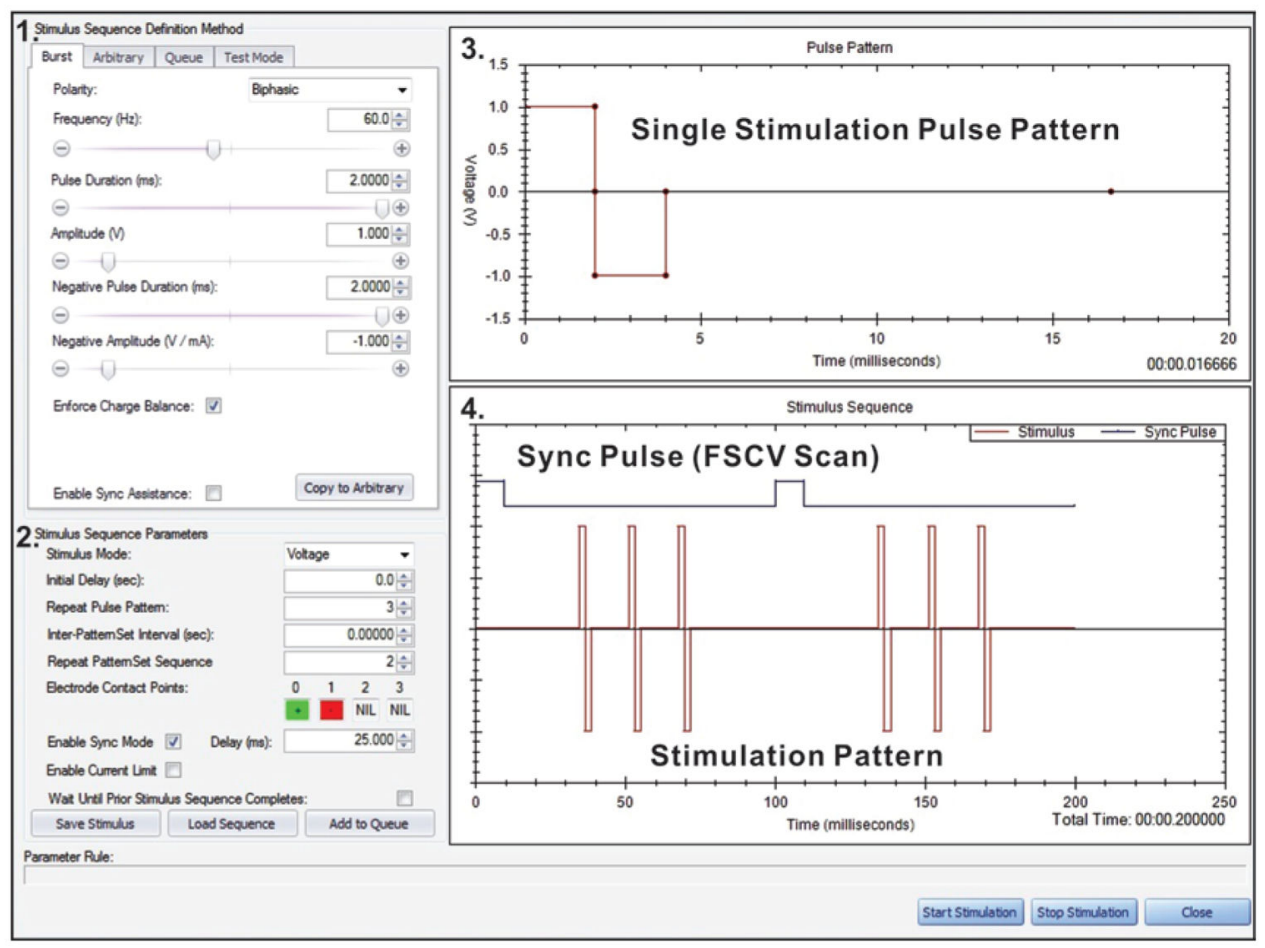

Fig. 2.

An example of the WincsWare user interface for defining stimulation parameters and synchronizing stimulation with FSCV. The user interface includes 1) the "Stimulus Sequence Definition Method" panel for selecting the characteristics of the stimulus pulses (for example, polarity, frequency, pulse duration, and amplitude); 2) the "Stimulus Sequence Parameters" panel for selecting the stimulus mode and presentation characteristics of the stimulus pulses (for example, initial delay, pulse pattern settings, and electrode contact); 3) the "Pulse Pattern" display in which a single pulse pattern can be user-adjusted via computer mouse; and 4) the "Stimulus Sequence" chart, depicting an overview of the user-selected stimulus pulse train in relation to the sync pulse from WINCS. The sync pulse indicates the end of an FSCV scan. The software facilitates interleaving stimulus pulses with FSCV scans. 


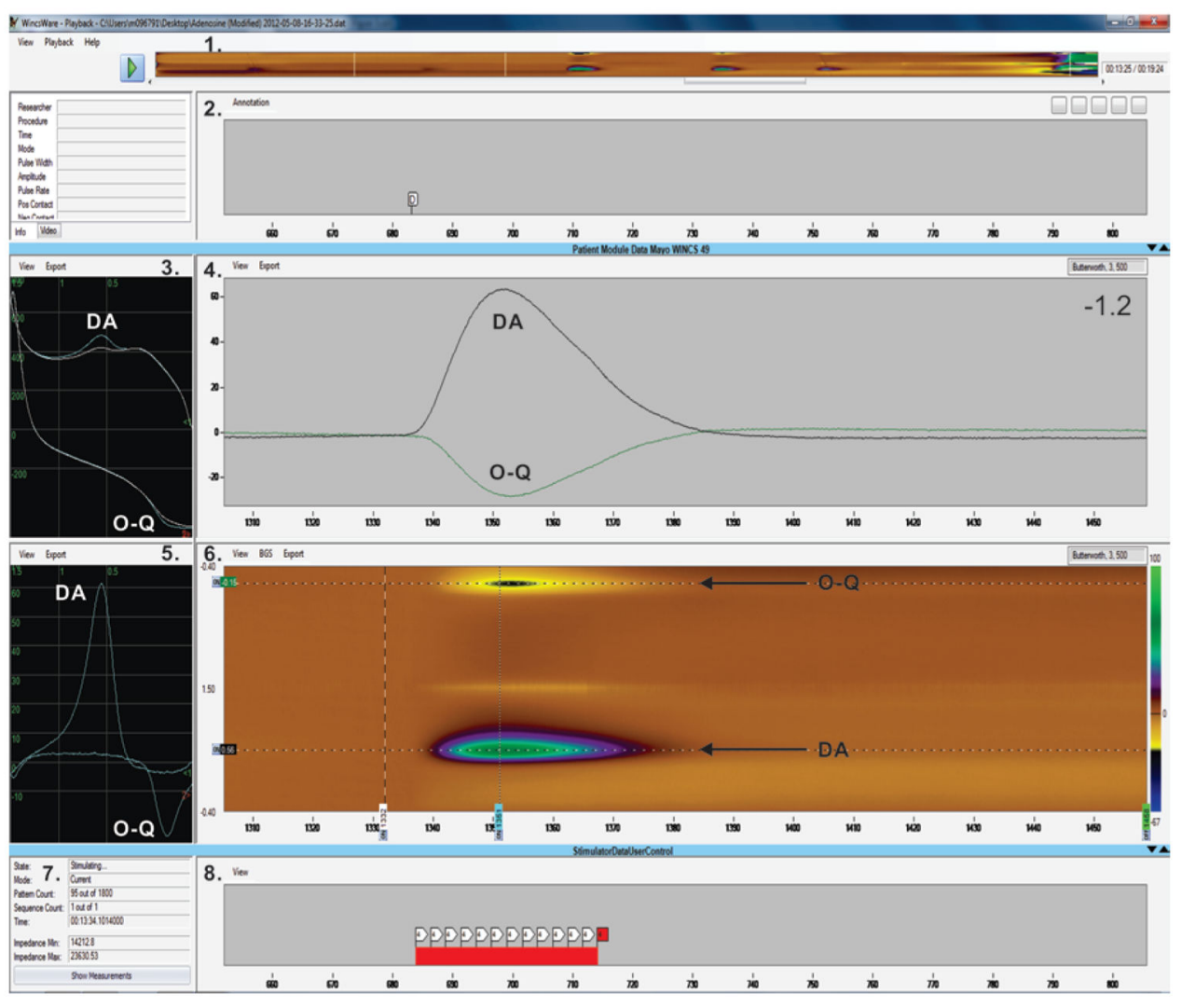

Fig. 3.

An example of the WincsWare user interface displaying acquired data in nearly real time. The displayed elements include 1) a color plot depicting data acquired throughout a recording session; 2) a panel displaying user-entered annotations; 3 ) raw (not background-subtracted) voltammograms, plotting electrode current as a function of applied potential for an FSCV scan. The blue line shows dopamine (DA) oxidation and orthoquinone $(\mathrm{O}-\mathrm{Q})$ reduction peak currents superimposed on the white line of the background current; 4) a current-versus-time strip chart of electrode currents measured at 1 or more selected applied potentials (typically the oxidation and reduction potentials for the analyte of interest; in this case, oxidation of DA and reduction of O-Q); 5) a background-subtracted voltammogram, here showing peaks for DA oxidation (at +0.56 V) and O-Q reduction (at $-0.16 \mathrm{~V}) ; 6$ ) the color plot of electrode current (represented by horizontal color traces) as a function of applied potential (vertical axis) for a sequence of FSCV scans over time (horizontal axis), here showing DA oxidation (green trail at $+0.56 \mathrm{~V}$ ) and $\mathrm{O}-\mathrm{Q}$ reduction back to DA (yellow and black trail at $-0.15 \mathrm{~V}$ ); 7) stimulation status information, including pattern and sequence count, elapsed time of stimulation, stimulating electrode impedance (calculated on the basis of measured stimulus current and voltage); and 8) a strip chart displaying intervals of stimulation. In panels 3 and 5, <1 indicates a forward direction $(-0.4 \mathrm{~V} \rightarrow+1.5 \mathrm{~V})$ and $<2$ indicates a backward direction $(+1.5 \mathrm{~V} \rightarrow-0.4 \mathrm{~V})$. The units on the $\mathrm{x}$ axes in panels $2,4,6$, and 8 are seconds, and in panels 3 and 5 they are volts. The units on the y axis in panels $3-5$ are nA and in panel 6 they are volts. 
A
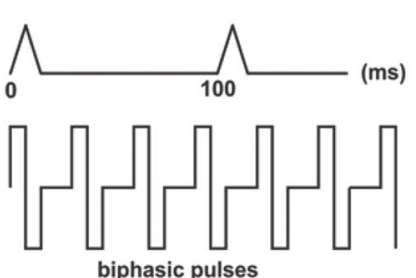

biphasic pulses

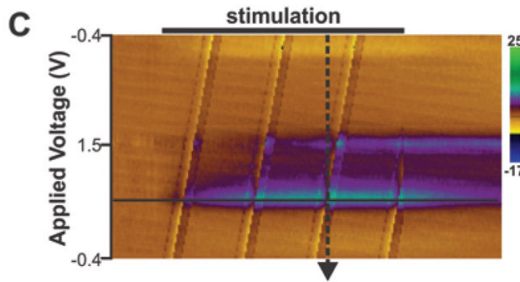

E

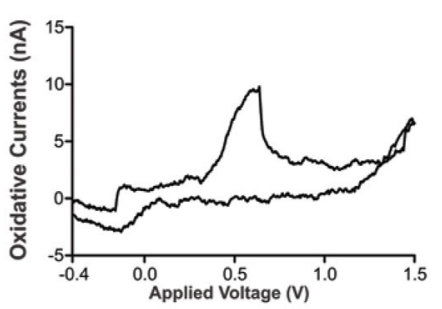

G

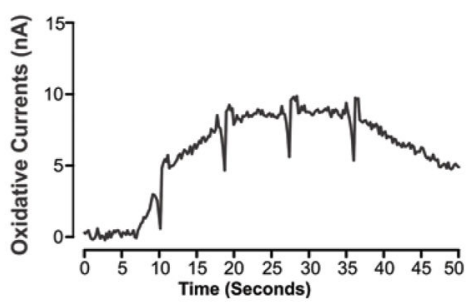

B

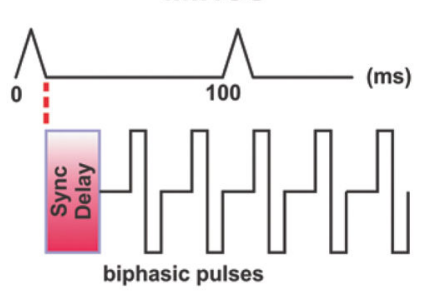

D

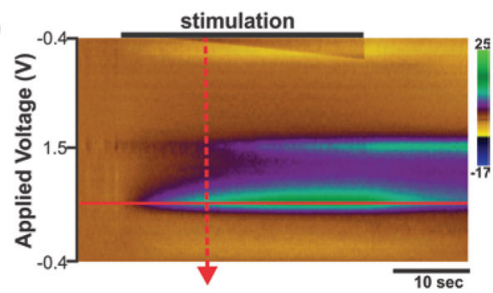

F

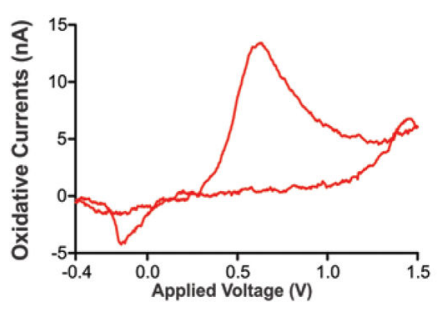

H

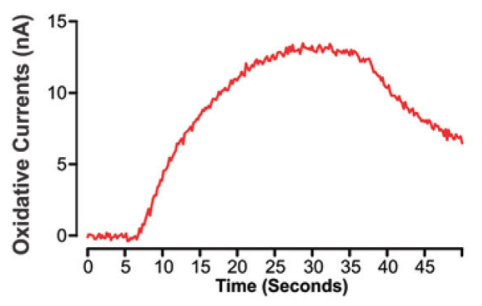

Fig. 4.

Elimination of stimulus pulse artifacts using MINCS-WINCS synchronization. A and B: Diagrams comparing the stimulus pulse sequences provided by a conventional stimulator (left) and MINCS (right) in relation to FSCV scans (pyramidal waveforms). Optically synced with WINCS, MINCS eliminates stimulus pulse interference with FSCV measurements by imposing a delay after every scan. C and D: Comparison of color plots of striatal dopamine release acquired in vivo from MFB stimulation (30-second stimulation at 60-Hz, 200- $\mu \mathrm{A}, 2-\mathrm{msec}$ biphasic pulse duration) in the anesthetized rat with (right) and without (left) stimulus pulse synchronization. Stimulus pulse artifacts are readily apparent as repeating diagonal bands in the color plot (left). E and F: Cyclic voltammograms recorded at the peak of stimulation-evoked dopamine release (right; dashed red arrow in D) and coincident to stimulus pulse interference (left; dashed black arrow in C). Note the marked distortion of the voltammogram recorded during the occurrence of a stimulus pulse. $\mathbf{G}$ and $\mathbf{H}$ : Time courses of stimulation-evoked changes in dopamine oxidation current extracted from each respective color plot record at the applied voltage of $+0.6 \mathrm{~V}$ (right, solid red line in D; left, solid black line in C). Stimulus pulse artifacts (negative-going spikes in current) were readily apparent using a conventional stimulator, while entirely absent using MINCS. 

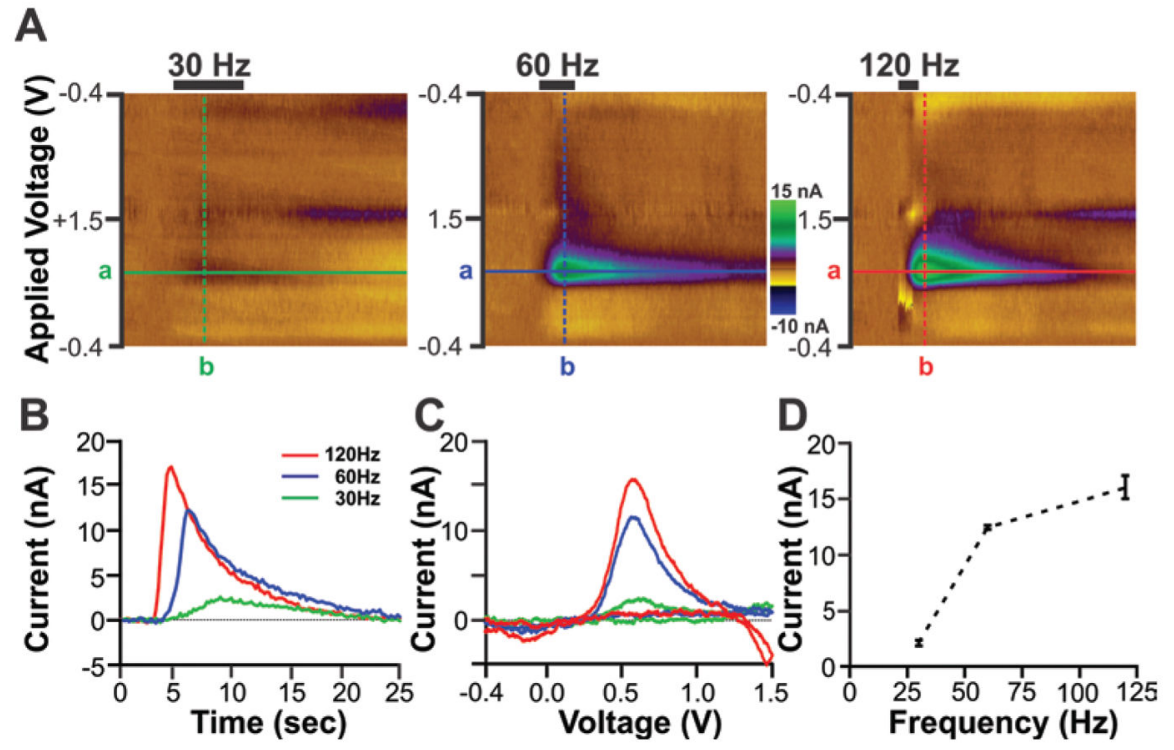

Fig. 5.

In vivo functionality tests of the integrated MINCS-WINCS devices showing frequency-dependent striatal dopamine release evoked by MFB electrical stimulation in anesthetized rats. A: Color plots obtained in response to electrical stimulation delivered at $30 \mathrm{~Hz}$ (left), $60 \mathrm{~Hz}$ (center), and $120 \mathrm{~Hz}$ (right). The number of pulses (120), stimulation intensity (200 $\mu \mathrm{A}$ ), and pulse duration $(2 \mathrm{msec})$ for the biphasic stimulation train were the same for each test frequency. The FSCV triangle waveform was applied from $-0.4 \mathrm{~V}$ to $+1.5 \mathrm{~V}$ with dopamine oxidation peak currents readily apparent at $+0.6 \mathrm{~V}$. B: Time course of changes in dopamine oxidation current in response to 30-, 60-, and 120-Hz electrical stimulation. These changes in dopamine oxidation currents were detected at the applied voltage $(+0.6 \mathrm{~V})$ shown in each color plot (line a). C: Voltammograms of dopamine oxidation and reduction after subtraction of prestimulation background current. These voltammograms were extracted at the time corresponding to maximal increases in dopamine oxidation current (line b). D: Stimulation frequency-dependent striatal dopamine release showing an exponential increase in mean \pm SEM peak dopamine oxidation current as a function of increasing stimulation frequency. Note that the data shown in panels A-C are from a representative animal and data are the mean \pm SEM of 3 stimulations/frequency in the same animal. 